\section{A 67-year-old woman with knee pain}

\author{
Alistair R. Demcoe MD, Eric R. Bohm MSc MD
}

A 67-year-old woman with a body mass index of 33 presents with a year-long history of worsening medial pain in both knees. Her symptoms are aggravated by activity and regularly interfere with many of her usual activities. No other joints are symptomatic, and she hasn't experienced erythema or warmth in her knees. Examination of her knees shows tenderness in the medial joint line, mild effusions, normal range of motion with crepitus and intact ligaments. Her hips and back are normal on examination. Her medical history includes hypertension and type 2 diabetes mellitus.

\section{What is the likely diagnosis?}

The most likely diagnosis for this patient is osteoarthritis of the knees. The differential diagnosis includes pes anserine bursitis, spontaneous osteonecrosis of the knee and inflammatory arthropathy. Osteoarthritis is more common in women and older people; in addition, obesity puts this patient at increased risk for knee osteoarthritis. ${ }^{1,2}$ Brief morning stiffness, persistent knee pain, a decrease in function, crepitus, restricted movement and bony enlargement are clinical features and findings on physical examination that comprise the European League Against Rheumatism (EULAR) criteria for the diagnosis of osteoarthritis. ${ }^{2}$

\section{Are any investigations necessary?}

Plain radiography is the first-line imaging modality for the assessment of knee pain in this patient population. The EULAR group considers plain radiography (standing anteroposterior, standing semi-flexed posteroanterior, Merchant [skyline] and lateral views) the current gold standard for structural assessment of knee osteoarthritis. ${ }^{2}$ Magnetic resonance imaging is not required to make the diagnosis of osteoarthritis, ${ }^{2}$ nor is it helpful in making decisions about currently available interventions. ${ }^{1}$ Inappropriate use of magnetic resonance imaging is costly and can result in the detection and treatment of incidental meniscal tears. Degenerative meniscal tears are very common in patients with osteoarthritis and do not require operative treatment. ${ }^{1}$

\section{What initial treatment should be recommended?}

Several evidence-based clinical practice guidelines recommend the following initial interventions for the management of knee osteoarthritis: participation in a self-management program, strengthening exercises, low-impact exercises (aquatic or land-based), neuromuscular education and weight management. ${ }^{3-6}$

The Arthritis Self-Management Program was developed at Stanford University and is supported by The Arthritis Society. This widely used program is designed to help patients better understand their diagnosis and to encourage patients to take an active role in managing their arthritis and chronic pain. Meta-analyses and systematic reviews have shown that generalized strength training for the lower limbs and specific strength training for the quadriceps reduce pain effectively and improve physical function in osteoarthritis. ${ }^{4,5}$ A guideline from the American Academy of Orthopaedic Surgeons (AAOS) recommends neuromuscular education on the basis of several studies showing positive effects with kinesthesia, balance and proprioception training programs in patients with knee osteoarthritis. ${ }^{3}$

A guideline from the Osteoarthritis Research Society International (OARSI) recommends that a weight loss of $5 \%$ should be achieved within a 20 -week period, a rate of $0.25 \%$ per week, to be efficacious. ${ }^{4}$ A recent randomized controlled trial (RCT) stressed the importance of both diet and exercise in achieving weight loss and in managing knee oseteoarthritis. ${ }^{7}$

Patients randomly assigned to diet and exercise achieved more weight loss and had better physical health-related quality-of-life scores than patients assigned to exercise alone. Additionally, these patients had superior pain and function scores than patients assigned to either diet or exercise alone. ${ }^{7}$ The OARSI guideline also recommends use of a cane to help alleviate pain and improve function. ${ }^{4}$

\section{Does the patient require medication?}

When nonpharmacological intervention proves unsatisfactory, multiple guidelines recommend
Choosing Wisely Canada

In partnership with the Canadian Medical Association

Competing interests: None declared.

This article has been peer reviewed.

Correspondence to: Eric Bohm, ebohm@cjrg.ca

CMAJ 2014. DOI:10.1503 /cmaj.141129 
acetaminophen as a first-line agent for mild to moderate arthritis. Because of reports of gastrointestinal adverse events, elevated hepatic enzymes and overdose, the OARSI guideline recommends conservative dosing and treatment duration of acetaminophen. ${ }^{4}$ Although the OARSI guideline does not give exact parameters on this recommendation, the AAOS guideline indicates it may be prudent to restrict the over-the-counter dose of acetaminophen to $3000 \mathrm{mg}$ per day and reserve the $4000 \mathrm{mg}$ per day dose for prescriptions. ${ }^{3}$

Second-line agents include oral and topical nonsteroidal anti-inflammatory drugs (NSAIDs), selective cyclooxygenase- 2 inhibitors and topical capsaicin. ${ }^{6}$ Use of oral and topical NSAIDs received a strong recommendation in the AAOS guideline. ${ }^{3}$

For patients with osteoarthritis localized to one or both knees, the OARSI guideline recommends the use of local medications, including topical NSAIDs and corticosteroid injections (see below). ${ }^{4}$ This was especially emphasized in patients with coexisting medical comorbidities, which are very common in this patient population. ${ }^{4}$ Each medication carries unique safety and adverse-effect profiles, and therapies should be specific to each patient's individual risk factors and medical comorbidities.

\section{If initial treatment fails, what other options may be considered?}

Several options may be considered when initial treatment does not result in satisfactory symptom control. These include systemic medications (e.g., tramadol, duloxetine, opioids), intra-articular corticosteroid injections, medial compartmentunloading braces, physical modalities and manual therapy.

For refractory symptoms, guidelines recommend tramadol ${ }^{3,4,6}$ and duloxetine ${ }^{4,6}$ The AAOS recommendation for the use of tramadol is on the basis of five RCTs that showed outcomes in favour of the treatment group. ${ }^{3}$ Duloxetine is recommended by OARSI on the basis of a systematic review and an RCT that showed that the drug is efficacious and well tolerated for chronic pain associated with knee osteoarthritis. ${ }^{4}$ Whereas the use of intra-articular corticosteroid injections was deemed inconclusive in the AAOS guideline, ${ }^{3}$ the OARSI guideline supports their use because of two systematic reviews showing significant shortterm decreases in pain. ${ }^{4}$

The use of physical modalities (e.g., transcutaneous electrical nerve stimulation, ultrasound, therapeutic application of musically modulated electromagnetic fields) and manual therapy all received an inconclusive recommendation from the AAOS work group. ${ }^{3}$ These recommendations are supported by several other guidelines. ${ }^{4,6}$
Although some older systematic reviews suggested minor benefits for the use of physical modalities or were inconclusive, ${ }^{3,4}$ more recent randomized trials showed no additional benefits to the use of these modalities. ${ }^{4}$ Manual therapy received an inconclusive rating in the AAOS guideline based on a lack of adequate studies evaluating joint mobilization, joint manipulation, chiropractic therapy, patellar mobilization or myofascial release. ${ }^{3}$

In a Cochrane review, use of opioids for hip and knee arthritis was found to be effective for pain control and improvement in physical function; however, because of a high rate of adverse events, the authors did not recommend the routine use of opioids for the treatment of osteoarthritis. ${ }^{8}$ Most articles in this review combined data on hip and knee osteoarthritis. The AAOS group found no studies on opioids or pain patches for the treatment of knee osteoarthritis that met its inclusion criteria, and the group was unable to recommend for or against opioid use. ${ }^{3}$ Other guideline groups were also unable to make a recommendation regarding opioid use. ${ }^{4,6}$

Medial compartment-unloading braces received an inconclusive recommendation from the AAOS ${ }^{3}$ however, this intervention was supported by OARSI. ${ }^{4}$ The evidence for their use appears to be inconsistent. The AAOS recommendation was based on inconsistent findings from three moderate- to high-quality RCTs, with improvement in pain scores not always reaching statistical significance. ${ }^{3}$

With inconclusive or uncertain recommendations, both the AAOS and OARSI guidelines stress that clinical judgment and patient preference should have a substantial influencing role when deciding to use these treatment modalities for the management of knee osteoarthritis. ${ }^{3,4}$

\section{What treatments shouldn't be offered?}

There are many other treatments that have been used for osteoarthritis, including needle lavage for joints, lateral wedge insoles, acupuncture, glucosamine, chondroitin, intra-articular injections of hyaluronic acid, and arthroscopy. However, studies of these treatments have not consistently shown statistically significant or clinically important improvements, and their use is not routinely recommended in guidelines.

Needle lavage for joints was assessed in a 2010 Cochrane review, and the authors found no benefit in terms of pain relief or improvement in physical function. ${ }^{9}$ Neither the EULAR nor the AAOS guidelines recommend using lateral wedge insoles because of lack of efficacy in comparison to neutral insoles ${ }^{3,5}$ and reports of adverse events. ${ }^{5} \mathrm{~A}$ recent meta-analysis investigating the 
use of lateral wedge insoles for treatment of medial knee osteoarthritis failed to show a statistically significant or clinically important improvement in pain scores using the Western Ontario and McMaster Universities Osteoarthritis Index (WOMAC) when compared with a neutral insole. ${ }^{10}$ The Canadian Orthopaedic Association, as part of the Choosing Wisely Canada campaign, does not recommend the use of either joint lavage or lateral wedge insoles for the treatment of knee arthritis $^{11}$ (Box 1).

The AAOS guideline recommends against the use of acupuncture, glucosamine, chondroitin and hyaluronic acid injections for the management of osteoarthritis of the knee. ${ }^{3}$ Other guidelines, including the 2014 OARSI guideline, are less critical of these modalities; however, they also do not recommend their use. ${ }^{4,6}$

Acupuncture is not recommended by the AAOS based on 10 studies (five high strength and five moderate strength), most of which did not show statistically significant improvement, and if they did show improvement, it was not clinically significant. ${ }^{3}$ The uncertain recommendation in the OARSI guideline on acupuncture was based on a meta-analysis that found statistically significant, but not clinically significant, benefit in sham-controlled trials. ${ }^{4}$

Like the AAOS, the Canadian Orthopaedic Association, as part of the Choosing Wisely Canada campaign, recommends against the use of glucosamine or chondroitin in patients with symptomatic osteoarthritis of the knee (Box 1). The AAOS group based its recommendation on the analysis of 5 high-quality, 1 low-quality and 15 moderate-quality studies, which failed to show improvement in pain and function scores using WOMAC and pain scores using a visual analogue scale. ${ }^{3}$ The OARSI guideline was less critical than the AAOS guideline, but more specific. The OARSI guideline indicates that glucosamine and chondroitin are not appropriate for use in knee osteoarthritis as disease-modifying agents and, partly because of inconsistencies in results between industry-sponsored and independent trials, was uncertain about their role in pain relief. ${ }^{4}$ An older Cochrane review on the use of glucosamine in osteoarthritis showed that its use, when restricted to analysis of studies with adequate allocation concealment and not using a specific brand, failed to show a benefit in WOMAC pain or function scores. ${ }^{12}$

The AAOS group based its recommendation on intra-articular hyaluronic acid injections on 14 studies (3 high-strength and 11 moderatestrength studies). Meta-analyses of pain, function and stiffness scores using WOMAC subscales all found statistically significant treatment effects with use of hyaluronic acid injections; however, none of the improvements met the AAOS group's threshold for minimal clinically important improvement. ${ }^{3}$ The OARSI's uncertain recommendation was based on two systematic reviews and an RCT that provided inconsistent conclusions and conflicting results. ${ }^{4}$

Over the past decade, there has been increasing evidence against the use of arthroscopy in the management of knee osteoarthritis. The current AAOS guideline reflects this and strongly recommends against the use of arthroscopy in the management of knee osteoarthritis. ${ }^{3}$ This recommendation was based on three RCTs (two with moderate strength and one with strong strength) that failed to show any clinical benefit, as well as on the risk associated with surgical intervention. ${ }^{3}$ This recommendation did not apply to patients with a primary diagnosis of meniscal tear and concomitant knee osteoarthritis. In this patient population, the AAOS found insufficient evidence to recommend for or against arthroscopic partial meniscectomy and rendered an inconclusive recommendation. ${ }^{3}$

\section{The case}

Plain radiography confirmed the diagnosis of moderate osteoarthritis of the knees. The patient was referred to a self-management program through The Arthritis Society (www.arthritis.ca/ asmp) for control of her knee osteoarthritis. As an initial management plan, a low-impact exercise program, a weight-loss plan that included both diet and exercise, and an acetaminophen dose of $500 \mathrm{mg}$ every 6 hours as required was recommended. Should the initial management plan fail to provide sufficient symptomatic relief, a topical NSAID would be a safe and generally effective addition, taking into consideration that the patient

\section{Box 1: Choosing Wisely Canada recommendations*}

Don't use needle lavage to treat symptomatic osteoarthritis of the knee for long-term relief.

- The use of needle lavage in patients with symptomatic osteoarthritis of the knee does not lead to measurable improvements in pain, function, 50-foot walking time, stiffness, tenderness or swelling.

Don't use glucosamine and chondroitin to treat symptomatic osteoarthritis of the knee.

- Both glucosamine and chondroitin sulfate do not provide relief for patients with symptomatic osteoarthritis of the knee.

Don't use lateral wedge insoles to treat symptomatic medial compartment osteoarthritis of the knee.

- In patients with symptomatic osteoarthritis of the knee, the use of lateral wedge or neutral insoles does not improve pain or functional outcomes. In addition, the possibility exists that those who do not use them may experience fewer symptoms from osteoarthritis of the knee.

*Source: Canadian Orthopaedic Association: Five things physicians and patients should question. Choosing Wisely Canada; 2014. Available: www.choosingwiselycanada.org/ recommendations/canadian-orthopaedic-association-2. 
has isolated knee osteoarthritis and comorbidities of hypertension and diabetes. Orthopedic referral will be initiated for consideration of knee replacement in the future if her pain and resultant functional limitations are inadequately controlled with comprehensive nonoperative management and she is willing to consider surgery.

\section{References}

1. Bennell KL, Hunter DJ, Hinman RS. Management of osteoarthritis of the knee. BMJ 2012;345:e4934.

2. Zhang W, Doherty M, Peat G, et al. EULAR evidence-based recommendations for the diagnosis of knee osteoarthritis. Ann Rheum Dis 2010;69:483-9.

3. Treatment of osteoarthritis of the knee. 2nd ed. Rosemont (IL): American Academy of Orthopaedic Surgeons; 2013.

4. McAlindon TE, Bannuru RR, Sullivan MC, et al. OARSI guidelines for the non-surgical management of knee osteoarthritis. Osteoarthr Cartil 2014;22:363-88.

5. Fernandes L, Hagen KB, Bijlsma JW, et al. EULAR recommendations for the non-pharmacological core management of hip and knee osteoarthritis. Ann Rheum Dis 2013;72:1125-35.

6. Nelson AE, Allen KD, Golightly YM, et al. A systematic review of recommendations and guidelines for the management of osteoarthritis: The Chronic Osteoarthritis Management Initiative of the U.S. Bone and Joint Initiative. Semin Arthritis Rheum 2014;43:701-12.

7. Messier SP, Mihalko SL, Legault C, et al. Effects of intensive diet and exercise on knee joint loads, inflammation, and clinical outcomes among overweight and obese adults with knee osteoarthritis: the IDEA randomized clinical trial. JAMA 2013;310:1263-73.

8. Nüesch E, Rutjes AW, Husni E, et al. Oral or transdermal opioids for osteoarthritis of the knee or hip. Cochrane Database Syst Rev 2009;(4):CD003115

9. Reichenbach S, Rutjes AW, Nuesch E, et al. Joint lavage for osteoarthritis of the knee. Cochrane Database Syst Rev 2010;(5): CD007320.

10. Parkes MJ, Maricar N, Lunt M, et al. Lateral wedge insoles as a conservative treatment for pain in patients with medial knee osteoarthritis: a meta-analysis. JAMA 2013;310:722-30.

11. Canadian Orthopaedic Association. Five things physicians and patients should question. Choosing Wisely Canada in partnership with the Canadian Medical Association. Available: www .choosingwiselycanada.org/wp-content/uploads/2014/03/2014-03 -28 EN-COA-List-CLEAN.pdf (accessed 2014 Aug. 26).

12. Towheed TE, Maxwell L, Anastassiades TP, et al. Glucosamine therapy for treating osteoarthritis. Cochrane Database Syst Rev 2005;(2):CD002946.

Affiliations: Concordia Hip \& Knee Institute (Demcoe, Bohm), Winnipeg, Man.; Department of Surgery, Section of Orthopaedic Surgery (Demcoe), University of Manitoba; Section of Orthopaedic Surgery, Department of Surgery (Bohm), University of Manitoba, Winnipeg, Man.

Contributors: Alistair Demcoe selected the reference material, and drafted and revised the article. Eric Bohm conceptualized and revised the article. Both authors approved the final submission.

CMAJ is collaborating with Choosing Wisely Canada, with support from Health Canada, to publish a series of articles describing how to apply the Choosing Wisely Canada recommendations in clinical practice.

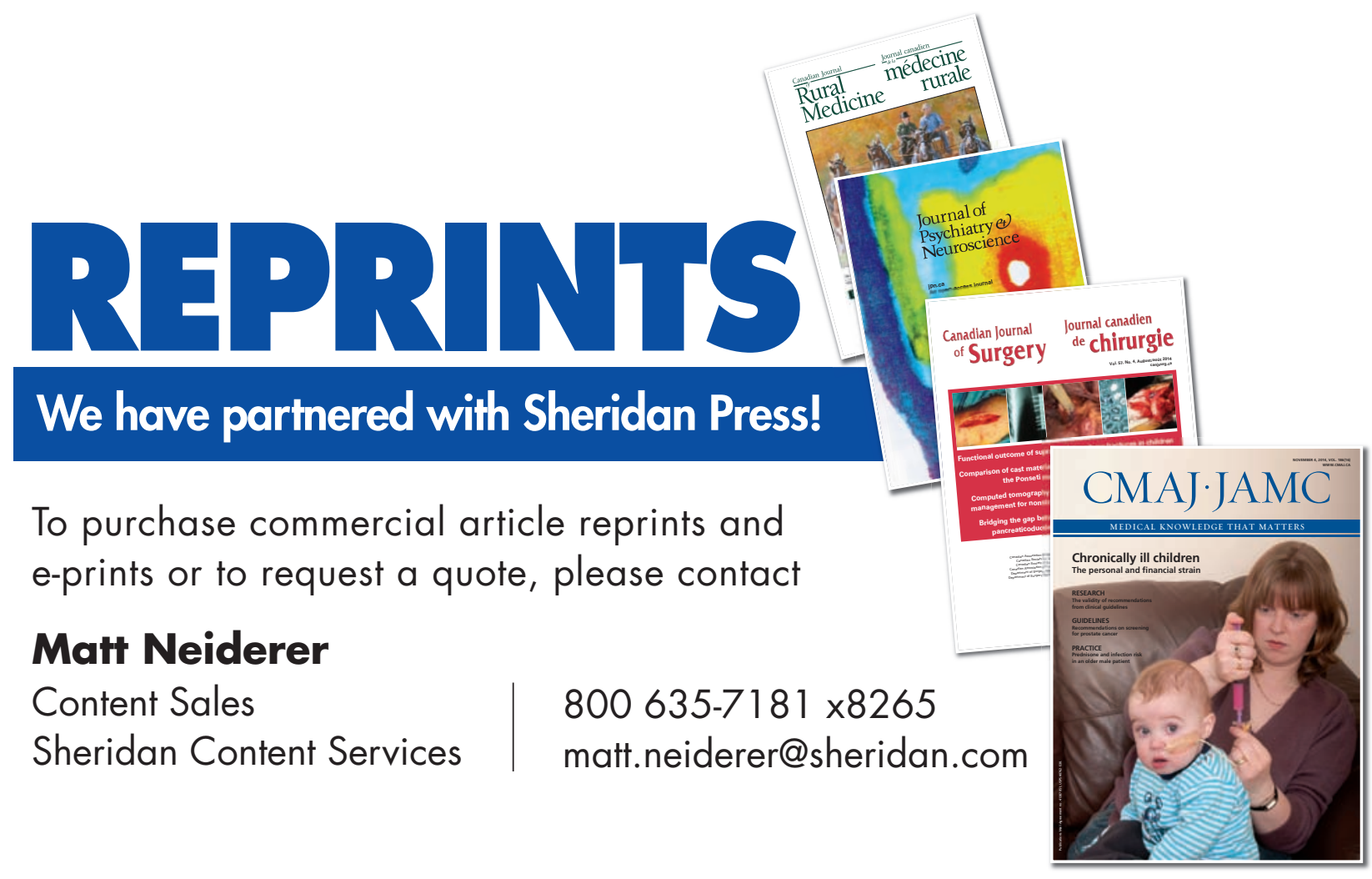

